\title{
A rare presentation of superficial, inter-muscular, cystic, extra- spinal schwannoma over upper back
}

\section{Srinjoy Saha}

Department of Plastic Surgery, Apollo Gleneagles Hospital, Kolkata, India

\section{Cite this article:}

Saha S. A rare presentation of superficial, intermuscular, cystic, extra-spinal schwannoma over upper back. Neurologico Spinale Medico Chirurgico. 2020.3(3): 103-106.

DOI: I0.36444/nsmc.v3i3.120

Corresponding author:

Dr Srinjoy Saha, MBBS, MS, MRCS, MCh (Plast)

Apollo Gleneagles Hospital,

Day Care Building $2^{\text {nd }}$ Floor,

58 Canal Circular Road, Kolkata 700054, India.

Phone: +9l 9874633896

ss@medi.ac

\section{Abstract}

Schwannomas are rare tumours arising from peripheral nerve sheath and are usually related to the spinal cord and spinal nerves. Contrast magnetic resonance imaging (MRI) usually helps in establishing a pre-operative diagnosis. Rarely, their manifestation may be surprisingly new. Here, a 44-year-old lady came to our clinic with a painless asymptomatic progressively enlarging swelling over her upper back. It was fluctuant, with absent neural signs and symptoms. MRI showed a benign, purely-cystic, superficial-intermuscular, extra-spinal swelling near the upper thoracic vertebrae. However, classical diagnostic signs of schwannoma were absent. Complete surgical excision was performed with smooth dissection through a well-defined plane between the lesion and surrounding muscles. A $6.5 \times 5.0 \times 2.5 \mathrm{~cm}$ oval lesion with a glistening whitish-grey capsule was excised, and the deep wound was reconstructed in multiple layers. Interestingly, it was not attached to any identifiable nerves. Histopathology showed typical hallmarks like Antoni A regions and Verocay bodies. Positive S-100 staining during immunohistochemistry established its diagnosis as schwannoma. The postoperative one-andhalf-year follow-up period was uneventful. Cystic schwannomas can surprise and confuse clinicians by arising anywhere in the body and with atypical manifestations. Surgeons need to consider it in the differential diagnoses of any undiagnosed slowly-growing swelling, including purely-cystic ones and perform careful surgical dissection to avoid any inadvertent nerve damage.

Keywords: Cystic schwannoma, extraspinal cyst, superficial intermuscular cyst, magnetic resonance imaging, surgical excision

\section{Introduction}

Schwannomas (also called neurilemmomas) are tumours arising from the Schwann cells, which sheathe, support and protect the peripheral nerves by warping around axons. Usually, they are benign encapsulated solid tumours, and rarely cancerous. ${ }^{1}$ On magnetic resonance imaging (MRI), classical signs like 'split-fat sign' and 'target sign' may be present. Degenerative changes like hyalinisation, haemorrhage, calcification and cyst formation may occur. ${ }^{2}$ Fine needle aspiration cytology (FNAC) from the solid part of a schwannoma usually establishes the diagnosis 
pre-operatively. Purely cystic schwannomas are rarely encountered.

Described over here is an unusual presentation of a schwannoma. After searching previous literature, none of the schwannomas reported earlier had an identical manifestation as in this case with the following atypical features:

- Clinically asymptomatic without any associated neural signs or symptoms,

- Completely cystic without any underlying firmness,

- Lying in between the superficial back muscles,

- Not related to any known or suspected nerve course,

- Located far away from the spinal cord and dorsal rami,

- Situated over the upper dorsum (interestingly not over the cervical or lumbar regions, which are more classically affected and raise suspicion).

- Contrast MRI did not reveal any of the classical signs, and

- $\quad$ FNAC was inconclusive.

\section{Case report}

A 44-year-old woman came to our clinic with an asymptomatic slowly progressive swelling over her upper back. She first noticed the swelling incidentally, small as a lemon, about four years ago. Her medical history was unremarkable, and she did not have any sensory or motor changes. Clinical examination revealed a spherical non-tender swelling in the paraspinal area over the upper back, sized $7.0 \times 6.5 \mathrm{~cm}$ (Figure 1A). Fluctuation was present on palpation, but thrill was absent. There were no other associated symptoms and no tenderness on spinal palpation. MRI revealed a well-defined, smooth-margin, oval-shaped swelling in the superficial-intermuscular plane. It lay underneath the trapezius, displacing the rhomboid and serratus posterior superior, and was superficial to the erector spinae muscles (Figures 2A,2B). While extending deep and medially towards the right side of the spinous process, it stayed far-off the dorsal spinal rami. There was a clear-cut plane between the cyst and surrounding muscles, with no local invasion (Figures 2C,2D). Relationship with any identifiable nerve was absent, nor was it related to any nerve course. Central part appeared necrotic with blood-fluid levels, with calcifications in the wall (Figure 2A,2C). Contrast-enhanced T1-weighted images showed ring-like capsular enhancement (Figures 2B,2D). Ultrasound-guided FNAC was performed, but the results were inconclusive. The pre-operative diagnosis was that of a benign, purely-cystic, superficial, intermuscular, extra-spinal swelling. The patient was scheduled for surgical excisional biopsy.

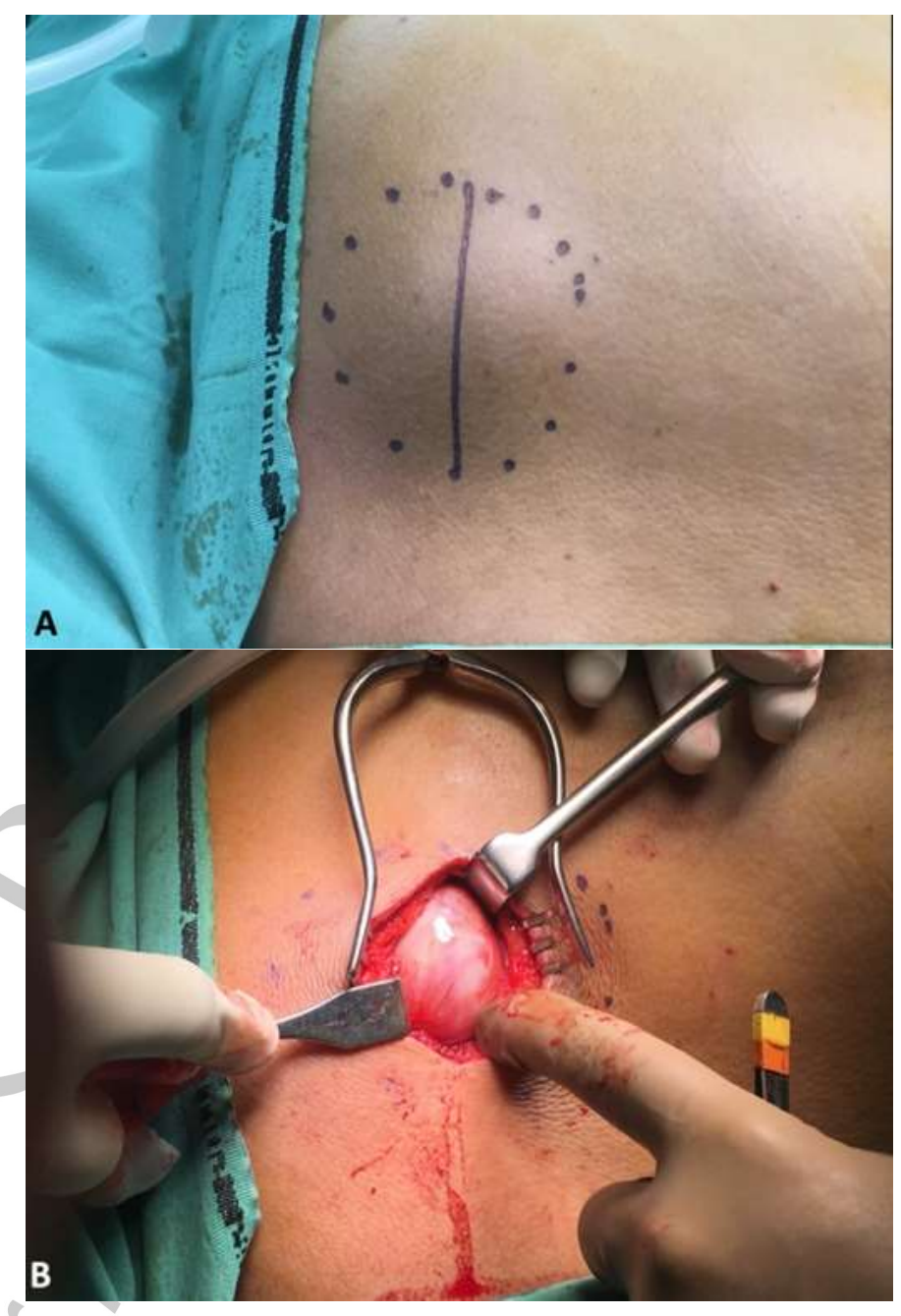

Figure 1. (A) A fluctuant swelling over the upper back measured $7.0 x 6.5 \mathrm{~cm}$. (B) Intra-operatively, incising the deep fascia and retracting the trapezius revealed a well-defined lesion covered with a glistening whitish capsule.

After surgical exploration, incising the fascia and retracting the trapezius helped reach the cystic swelling underneath, covered with a glistening whitish-grey capsule (Figure 1B). It extended inferiorly and medially deep towards the spine, displacing the rhomboid and serratus posterior superior. Lying superficial to the erector spinae muscles, it extended towards the right spinous processes of upper thoracic vertebrae, but far-off from the spine or spinous ligaments (Figure 3A). Dissection proceeded effortlessly through a well-defined plane between the swelling and the surrounding muscles. 


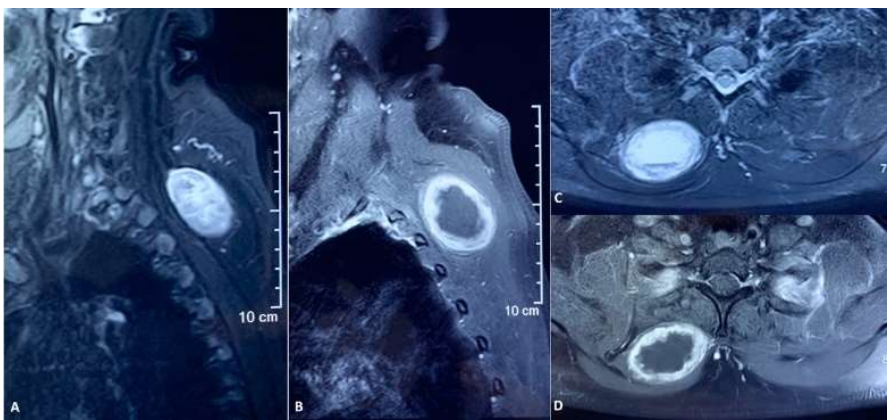

Figure 2. (A) MRI shows the lesion lying superficial to the erector spinae muscle in the region of the upper thoracic vertebrae. (B) T1-weighted image with rim-enhancement, signifying a purely cystic swelling. (C) MRI showed the lesion to be far-off from the spine in an inter-muscular plane superficially. (D) T1-weighted image shows rim-enhancement of the lesion and displacement of muscles over the upper back.

While a fascial pedicle attached its base to the underlying soft tissues, it was not adherent to any surrounding nerves. After complete surgical excision, the resulting wound was reconstructed in layers from deep to superficial. Gross examination of the specimen revealed a cystic swelling sized $6.0 \times 5.0 \times 2.5 \mathrm{~cm}$ (Figure 3B). The cut-section contained dark reddish-brown areas, signifying haemorrhagic changes inside. An encapsulated lesion with hyper and hypocellular areas was seen on histopathological examination. Cystic degeneration was present in the centre (Figure 4A). Bland oval to spindle-shaped cells was present. Hypercellular areas with nuclear palisading (oval-shaped Verocay bodies) were noted. Neither nuclear atypia nor increased mitotic activity was visible (Figure 4B). Immunohistochemistry was strongly positive for S-100, and negative for CD34, Cytokeratin-Pan (AE1/AE3), Smooth muscle actin, with Mib1 of 1\% (Figure 4C). Final diagnosis confirmed benign cystic schwannoma. Recovery during the postoperative period was uneventful, and the wound healed well with minimal scarring. There were no recurrences or other complications during the next one-and-half-year follow-up period.

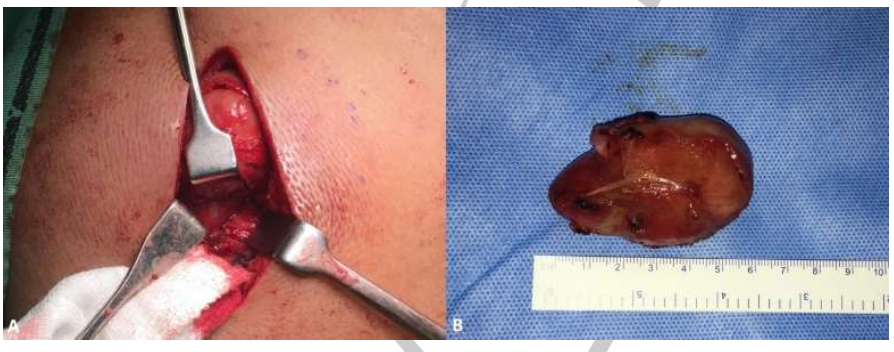

Figure 3. (A) The lesion was far-off from the spinous processes and spinous ligaments, and not attached to any specific nerve. (B) Complete surgical excision revealed a cystic swelling sized $6.0 \times 5.0 \times 2.5 \mathrm{~cm}$.

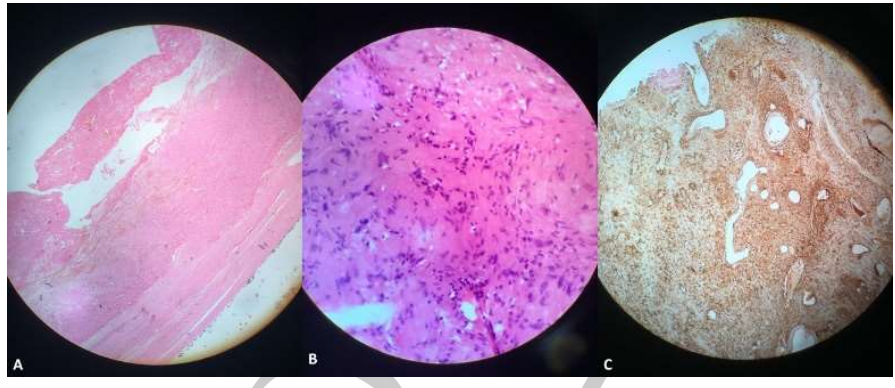

Figure 4. (A) Hematoxylin \& Eosin staining of the specimen shows the capsule of a cystic swelling under $4 x$ scanner view. (B) Oval-shaped Verocay bodies with characteristic nuclear palisading, classical of schwannoma, was identifiable on 40x high-power fields. (C) S-100 positivity was seen on immunohistochemistry, establishing the diagnosis of schwannoma.

\section{Discussion}

Neurogenic tumours comprise only about $5 \%$ of all benign softtissue tumours. Differential diagnoses of extra-spinal swellings include schwannoma, neurofibroma, meningioma, ependymoma, sarcoma, abscess, lymphoma, and osteoma amongst others. ${ }^{3}$ Schwannomas are most commonly found intra-durally. They may occur at any level of the spinal cord axis, including as a dumb-bell shaped tumour with both spinal cord axis, including as a dumb-bell shaped tumour with both intra-dural and extra-dural components. ${ }^{4} \mathrm{~A}$ superficial inter-muscular presentation of a purely-cystic, extra-spinal schwannoma has not been reported till now.

Amongst the common benign nerve sheath tumours, neurofibromas are located centrally and are inseparable from the originating nerve. In direct contradiction, schwannomas are located eccentrically over its originating nerve sheath. ${ }^{5}$ Typical schwannomas are well-encapsulated and solid, with membranes composed of a thin fibrous capsule. Degenerations occur within long-standing tumours, including hyalinisation, haemorrhage, calcification and cyst formation. ${ }^{6}$ Most commonly affected regions are head and neck $(25 \%-45 \%)$ with the lateral neck being the most affected region. Manipulation of the swelling sometimes produces paraesthesia or 'shocks' in the distribution of the affected nerve. ${ }^{7}$ Greater mobility from side-to-side compared to longitudinal is found in benign neurogenic tumours.

MRI is useful for pre-operative diagnosis. It may show characteristic 'split-fat sign', wherein a rim of fat surrounds the lesion. 'Entry-and -exit' signs of nerve roots entering and exiting the tumour are commonly visible at both ends of the fusiform mass. 'Target' sign is seen when the lesion shows a thin peripheral -hyperintense rim and a central lower-signal intensity on T2-weighted images. Signs of degeneration like cyst formation, haemorrhage (blood-fluid levels), and calcification may be identifiable. ${ }^{8}$

Classical histopathological characteristics of schwannoma 
include Antoni A and B lesions and Verocay bodies. Antoni A lesions occur in densely compact regions, where spindled cells with elongated nuclei are arranged haphazardly like whorls or waves. Verocay bodies consist of parallel rows of palisading elongated nuclei around a central mass of eosinophilic cytoplasm. Antoni B lesions, considered degenerative forms of type A, comprise loose pale hypocellular stromal tissues and lack classical bundled or palisading patterns. ${ }^{9}$ Usually, mitotic activities are absent in schwannoma; while malignant transformations are rare. Immunohistochemistry with S-100 staining establishes the neural origin of the tumour. ${ }^{10}$

\section{Conclusion}

Cystic extra-spinal schwannomas can surprise and confuse clinicians by mimicking a sizeable cystic swelling anywhere in the body. Surgeons need to be mindful and consider it in the differential diagnosis of any inconclusively diagnosed slowly-growing lesion, including cysts. Careful surgical dissection is warranted in all such cases to avoid transecting any nerves inadvertently, especially near the pedicle, to prevent any long-term iatrogenic complications. Careful, complete excision typically results in excellent postoperative outcomes.

\section{Acknowledgment}

Dr Sanjib Majumdar, MD; Dr Rajesh Ghosh, MD; and Dr Asra Nausheen Quadri, MD.

\section{References}

1. Kim DH, Murovic JA, Tiel RL, et al. A series of 397 peripheral neural sheath tumors: 30-year experience at Louisiana State University Health Sciences Center. J Neurosurg. 2005;102(2):246-

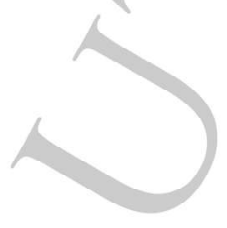

255. DOI:10.3171/jns.2005.102.2.0246

2. Crist J, Hodge JR, Frick M, et al. Magnetic Resonance Imaging Appearance of Schwannomas from Head to Toe: A Pictorial Review. J Clin Imaging Sci.2017;7:38. DOI:10.4103/jcis.JCIS_40_17

3. Kransdorf MJ. Malignant soft-tissue tumors in a large referral population: distribution of diagnoses by age, sex, and location. AJR Am J Roentgenol. 1995;164(1):129-134. DOI:10.2214/ajr.164.1.7998525

4. Montano N, D'Alessandris QG, D'Ercole M, et al. Tumors of the peripheral nervous system: analysis of prognostic factors in a series with long-term follow-up and review of the literature. J Neurosurg. 2016;125(2):363-371. DOI:10.3171/2015.6.JNS15596

5. MacCollin M, Chiocca EA, Evans DG, et al. Diagnostic criteria for schwannomatosis. Neurology. 2005;64(11):1838-1845. DOI:10.1212/01.WNL.0000163982.78900.AD

6. Knight DM, Birch R, Pringle J. Benign solitary schwannomas: a review of 234 cases. J Bone Joint Surg Br. 2007;89(3):382-387. DOI:10.1302/0301-620X.89B3.18123

7. Farschtschi SC, Mainka T, Glatzel M, et al. C-Fiber Loss as a Possible Cause of Neuropathic Pain in Schwannomatosis. Int J Mol Sci. 2020;21(10):3569. DOI:10.3390/ijms21103569

8. Zhang Z, Deng L, Ding L, et al. MR imaging differentiation of malignant soft tissue tumors from peripheral schwannomas with large size and heterogeneous signal intensity. Eur $J$ Radiol. 2015;84(5):940-946. DOI:10.1016/j.ejrad.2015.02.003

9. Rodriguez FJ, Folpe AL, Giannini C, et al. Pathology of peripheral nerve sheath tumors: diagnostic overview and update on selected diagnostic problems. Acta Neuropathol. 2012; 123(3): 295-319. DOI:10.1007/s00401-012-0954-Z

10. Louis DN, Ohgaki H, Wiestler OD, et al. editors. WHO Classification of Tumours of the Central Nervous System. 4th ed., Revised. Lyon: International Agency for Research Centre; 2016.

\footnotetext{
Articles published online under this model are officially published and can be cited and quoted using the DOI as the reference source. Neurologico Spinale Medico Chirurgico has a policy that changes will not be made after publication of an article without following accepted procedures for making corrections to the scientific record. The entire contents of the Neurologico Spinale Medico Chirurgico are licensed under the Creative Commons Attribution 4.0 International License. You are free to share - copy \& redistribute the material in any medium or format, adapt - remix, transform, and build upon the material for any purpose, even commercially. Under the following terms, you must give appropriate credit, provide link to the license, and indicate if changes were made. You may do so in any reasonable manner, but not in any way that suggests the licensor endorses you or your use.
} 